\title{
PREVALENCIA DE DEPRESIÓN POSPARTO EN PRIMIGESTAS Y MULTÍPARAS VALORADAS POR LA ESCALA DE EDIMBURGO
}

\author{
José Urdaneta M. MSc ${ }^{1}$, Ana Rivera S. ${ }^{1}$, José García I. PhD ${ }^{2}$, Mery Guerra V. PhD ${ }^{2}$, \\ Nasser Baabel Z. $P h D^{2}$, Alfi Contreras B. ${ }^{3}$ \\ 1 Programa de Obstetricia y Ginecología, Hospital Universitario de Maracaibo, Universidad del Zulia. ${ }^{2}$ Departamento de \\ Ginecología y Obstetricia, Universidad del Zulia. 3 Instituto Venezolano de los Seguros Sociales. Maracaibo, Venezuela.
}

\section{RESUMEN}

Antecedentes: Los trastornos depresivos representan un importante problema de salud pública, siendo más frecuente en las mujeres y con mayor riesgo durante el embarazo y puerperio. Objetivo: Comparar la prevalencia de depresión posparto en primigestas y multíparas atendidas en la División de Obstetricia y Ginecología del Servicio Autónomo Hospital Universitario de Maracaibo, Venezuela. Método: Se realizó una investigación comparativa con diseño no experimental, que incluyó una muestra de 100 mujeres en puerperio tardío, divididas en dos grupos pares de 50 sujetos según paridad, evaluadas mediante la Escala de Depresión Posparto de Edimburgo. Resultados: Los resultados demostraron una prevalencia del test positivo en el $91 \%$ de la muestra, con una prevalencia de depresión posparto confirmada por medio del DSM-IV del $22 \%$, presentando mayor frecuencia de ideación suicida las multíparas $(p<0,05)$. No hubo una asociación estadísticamente significativa entre paridad y depresión posparto. Conclusión: La población estudiada presenta una alta prevalencia de depresión postparto, sin diferencias en cuanto a su paridad.

\section{Palabras clave: Depresión posparto, escala de depresión posparto de Edimburgo, paridad}

\section{SUMMARY}

Background: Today the depressive upheavals represent an important public health's problem, being more frequent in the women and with greater risk during the pregnancy and puerperium. Objective: To compare the prevalence of postpartum depression in nulliparous and multiparous women taken care of Maternity "Dr. Armando Castillo Plaza" at the University Hospital of Maracaibo, Venezuela. Method: A comparative investigation with non experimental design was realized, it which included a sample of 100 women in later puerperium, divided in two even groups of 50 subjects according to her parity, evaluated by Edinburgh Postnatal Depression Scale. The results demonstrated a prevalence of the positive test in $91 \%$ of the sample, with a confirmed prevalence of postpartum depression by the DSM-IV of $22 \%$, presenting a highly frequency of suicidal ideas in the multiparous group $(p<0.05)$; also, don't found statistically significant association between parity and postpartum depression. Conclusión: The studied population show a highly prevalence of postpartum depression, without majors differences according to the parity.

KEY WORDS: Postpartum depression, Edinburgh postnatal depression scale, parity 


\section{INTRODUCCIÓN}

Los problemas emocionales durante el puerperio se conocen desde la antigüedad, ya en los primeros escritos de Hipócrates, 700 AC, los describía en detalle, así como también en los textos de Celcio y Galeno (1). En la actualidad, los trastornos depresivos representan un problema importante de salud pública, estos se observan con mayor frecuencia en la población femenina que en la masculina y parece ser sustancialmente mayor durante el embarazo y el puerperio que durante otras épocas del ciclo vital, especialmente en las embarazadas de bajos ingresos económicos (2). El trastorno depresivo es una enfermedad que afecta al cerebro, el ánimo y la manera de pensar, el cual es considerado como un desorden del talante, el cual constituye la forma más común de sufrimiento mental, millones de personas en todo el mundo, sin importar sus razas, nacionalidades o culturas sufren esta enfermedad (3).

Durante el embarazo y el puerperio se suceden una serie de cambios bioquímicos, psicológicos y sociales que ocasionan una mayor vulnerabilidad para la aparición de trastornos en la esfera psíquica de la mujer (4). La depresión posparto es entendida como la presencia de todo trastorno depresivo, sin síntomas psicóticos, que se manifiesta durante el primer año posparto (5). El cuadro clínico de la depresión posparto es similar al de los episodios depresivos observados en otras etapas de la vida, sin embargo, la depresión posparto posee características peculiares que justifican su consideración como entidad específica $(5,6)$.

La presencia de trastorno depresivo mayor, con frecuencia abreviado o llamado simplemente como depresión, durante el embarazo, incrementa en forma importante la morbilidad y mortalidad tanto para la madre como para el producto del embarazo (7); asociándose a conductas poco saludables como el omitir la atención prenatal o el uso de sustancias nocivas (tabaco, alcohol, sustancias ilegales) y constituyendo un factor de riesgo para intentos de suicidios y suicidio consumados (8).

Los desórdenes depresivos afectan al menos al $20 \%$ del sexo femenino y $12 \%$ de los hombres en algún momento durante su vida, siendo las mujeres doblemente susceptibles a sufrir depresión, y los síntomas generalmente se incrementan con la edad (3). Si bien los trastornos afectivos constituyen el segundo desorden más común dentro de las enfermedades mentales en todo el mundo, estimando la Organización Mundial de la Salud (OMS) que para la tercera década de este milenio sean la segunda causa general de discapacidad. El puerperio constituye entonces, una época en la cual es- tos trastornos son comunes, pero algunos, como la depresión posparto son poco reconocidos (9).

Resulta difícil determinar la prevalencia real de la depresión posparto, porque los estudios difieren extremadamente en lo que se refiere a metodología empleada y a las poblaciones analizadas, pero puede aceptarse que la frecuencia mundial de esta enfermedad fluctúa entre un 10 a $20 \%$ de las puérperas según algunos autores (5) o de un 10 a 15\% según otros $(4,10)$; con un rango entre 30 a 200 casos por cada 1000 nacimientos y con una estimación de una tasa de recurrencia entre 10 y $35 \%$ (1). La depresión posparto es un importante trastorno de la salud mental en la mujer, con una prevalencia tres veces más alta en los países en desarrollo que en los países desarrollados, siendo mas frecuente en las mujeres de nivel socioeconómico bajo $(4,5,10)$.

Llama la atención el hecho de que la depresión posparto sea habitualmente subdiagnosticada en centros de atención materno-infantil, pese a que su prevalencia supera la correspondiente a otras patologías obstétricas tales como preeclampsia, parto prematuro y diabetes gestacional, entre otras entidades (5). En este sentido, Evans y cols (10), expresan que la mayoría de las mujeres que experimentan depresión después del parto no tienen ayuda profesional, aún más, casi el $50 \%$ no tiene ayuda desde los familiares ni de los amigos. Por otro lado, las mujeres que tienen contacto con profesionales de la salud en sus controles posteriores al parto, no están dispuestas a manifestar sus problemas emocionales, en especial la depresión.

La presencia de síntomas mentales graves durante el embarazo y/o el postparto es importante por su morbimortalidad, consecuencias y posibles complicaciones, lo que ha llevado a que en los últimos años se haya intentado optimizar el diagnóstico y proveer tratamientos tempranos y eficientes para estos. Al respecto, se han diseñado varias escalas para el tamizaje de esta patología, entre los cuales se encuentra la escala de depresión posparto de Edimburgo (Edinburgh Postnatal Depression Scale, EPDS); es importante tener presente que la EPDS es un método de pesquisa para la depresión posparto, siendo el DSM-IV el método más exacto para el diagnóstico de esta entidad clínica. Según este último, el especificador de inicio en el posparto debe ser realizado a los trastornos que se inicien durante las cuatro semanas posteriores al parto y puede realizarse a episodios depresivos mayor, maniaco o mixto actual (o más reciente) de un trastorno depresivo mayor, trastorno bipolar I o trastorno bipolar II, o a un trastorno psicótico breve (11).

Por tanto, resulta fundamental y muy conveniente detectar esta patología entre las puérperas, 
para lo cual pudiesen aplicarse diversas técnicas de tamizaje que permitan identificar a aquellas madres con mayor necesidad de una evaluación diagnóstica más completa. El reconocimiento de la depresión posparto en la atención médica primaria es pobre, sobre todo si se considera el aumento que la depresión ha experimentado en toda la sociedad (10). Estas situaciones y el hecho de la omisión de la asistencia sanitaria puede originar trastornos en la interacción entre las madres y sus recién nacidos (12), tales como alteraciones psicológicas a corto y largo plazo en los hijos y un mayor riesgo de separaciones y divorcios (4).

El objetivo de esta investigación es comparar la depresión posparto en primigestas y multíparas atendidas en la Maternidad "Dr. Armando Castillo Plaza", de la División de Obstetricia y Ginecología del Servicio Autónomo Hospital Universitario de Maracaibo.

\section{PACIENTES Y MÉTODO}

Se procedió a efectuar una investigación de tipo comparativa y aplicada, adecuada a los propósitos del diseño no experimental, en donde se estudió la variable depresión posparto, describiendo su relación con factores de riesgo en primigestas y multíparas embarazadas. Las unidades de análisis estaban representadas por la totalidad de puérperas atendidas en la Maternidad "Dr. Armando Castillo Plaza", sede de la División de Ginecología y Obstetricia del Hospital Universitario de Maracaibo; de la cual se tomó una muestra de tipo no probabilística e intencionada, conformada por 100 mujeres puérperas atendidas en este centro asistencial durante el año 2009.

Esta muestra fue separada en dos grupos de 50 sujetos cada uno, de acuerdo con su paridad, fijándose como criterios para su inclusión los siguientes: (a) puerperio tardío, (b) edad mayor de 19 años y menor de 35 años, (c) ausencia de enfermedades psiquiátricas previamente diagnosticadas, y (d) deseo voluntario de participar en la investigación. Asimismo, se excluyeron a aquellas madres que fuesen anafalbetas o con discapacidad visual, debido a las características del instrumento empleado, así como a las mujeres cuyos embarazos hubiesen culminado en muertes perinatales o con productos malformados.

Se procedió a utilizar la técnica de la observación indirecta, mediante la utilización de un cuestionario autoadministrado, la Escala de Edinburgo para Depresión Posparto (EPDS), instrumento de auto reporte, el cual ha sido validado en múltiples países, estimándosele una sensibilidad cercana al $100 \%$ y una especificidad de $82 \%$ para evaluar la intensidad de la depresión durante el posparto. Este instrumento consta de 10 ítems con cuatro opciones de respuesta cada uno; las opciones tienen un puntaje que varía de 0 a 3 puntos, de acuerdo a la severidad de los síntomas, de modo que al final de la prueba, el puntaje varía entre 0 a 30 puntos (4).

Asimismo, se utilizó la técnica de la entrevista estructurada, resguardando la privacidad y confidencialidad de la paciente; para ello, se diseñó un guión de entrevista, conformado por diecinueve preguntas, diseñadas para la identificación de los factores de riesgo asociados a depresión posparto.

Para la ejecución de esta investigación, se procedió a seleccionar en las salas de maternidad a las puérperas de acuerdo con los criterios previamente establecidos, dividiéndose en dos grupos pareados según su paridad. Estas pacientes fueron citadas para su valoración posparto antes de las 6 semanas siguientes a la resolución del embarazo. A las puérperas se les solicitó su consentimiento informado de participación en el estudio, asimismo se registraron los siguientes datos: edad cronológica, paridad y tiempo de puerperio. Luego se les aplicó la EPDS, previa explicación de la misma; las mujeres participantes completaron el cuestionario mientras estaban sentadas solas en un consultorio. Seguidamente, se efectúo una consulta médica integral posparto, en donde se procedió a pesquisar los diferentes factores de riesgos mediante la entrevista clínica; para lo cual se siguió un guión de entrevista.

Las pacientes quienes obtuvieron un tamiz positivo fueron referidas al Servicio de Salud Mental, donde especialistas en el área de Psiquiatría por medio de una entrevista semiestructurada de acuerdo con el Manual de Diagnóstico y Estadísticas de Trastornos Mentales (DSM IV) confirmaron la presencia de depresión posparto.

Posteriormente, los datos obtenidos de la aplicación de estos instrumentos, fueron organizados, recopilados, e introducidos en una base de datos y por medio del Paquete Estadístico para Ciencias Sociales (SPSS), versión 11.0.1, se aplicó un tratamiento estadístico de tipo descriptivo; determinándose medidas de tendencia central (medias y desviación estándar) y realizándose la comparación de los resultados obtenidos entre los dos grupos evaluados, mediante la prueba del $x^{2}$ y la $U$ de Mann-Whitney, para grupos independientes según distribución de variables estudiadas. 


\section{RESULTADOS}

En la Tabla I se presentan algunas características demográficas y clínicas de la población estudiada. La edad de las pacientes primigestas fue en promedio 23 años (rango: 19-28 años), mientras que el grupo de pacientes multíparas la edad promedio fue de 27 años (rango: 21-33 años), evidenciándose que el grupo de pacientes primigestas mostró una tendencia a edades menores que el grupo de pacientes multíparas. También se evaluó el tiempo posterior al parto de la realización de la evaluación, evidenciándose que en ambos grupo las pacientes se encontraban en el puerperio tardío, alrededor de las 5 semanas. A su vez, el control prenatal, evaluado según el número de consultas efectuadas, fue adecuado en los dos grupos, con medias entre 6 y 7 consultas.

\section{Tabla I}

\section{RESULTADOS NEONATALES: GRUPO EN ESTUDIO Y CONTROL}

\begin{tabular}{lcc}
\hline Análisis descriptivo & $\begin{array}{c}\text { Primigestas } \\
\text { Media } \pm \mathrm{DE}\end{array}$ & $\begin{array}{l}\text { Multíparas } \\
\text { Media } \pm \mathrm{DE}\end{array}$ \\
\hline Edad & $23,1 \pm 5,3$ & $26,6 \pm 6,3$ \\
Paridad & - & $2,7 \pm 0,8$ \\
Número de & $7,8 \pm 1,1$ & $6,1 \pm 1,4$ \\
controles prenatales & & \\
Tiempo de & $5,1 \pm 1,5$ & $5,3 \pm 1,4$ \\
puerperio (semanas) & & \\
\hline
\end{tabular}

Como puede observarse en la Tabla II, la EPDS en su evaluación por ítems, evidenció tanto en las pacientes primigestas como en las multíparas, para los ítems que midieron el indicador disforia, las alternativas de respuesta mostraron una tendencia mayor a la alternativa 0 para los ítems 1 y 2 ; y los ítems 7,8 y 9 mostraron respuestas con tendencia a las alternativas 2 y 3 , considerando que las alternativas favorables para la evaluación integral del test son las que se acercan a 0 . Para el indicador ansiedad se observó que la alternativa considerada por las pacientes de ambos grupos, en sus respuestas fueron la 0 y 2 con una media de 1 para el ítems 4 , y para el ítems 5 la alternativa que mostró una tendencia mayor fue la 3. Para el indicador sentimiento de culpa las alternativas que mostraron una mayor tendencia en las respuestas fueron las alternativas 1 y 3 , evidenciando promedios mayores de 1,5 para estos ítems. El indicador dificultad de concentración mostró que para el ítems que lo mide, la respuesta con mayor frecuencia escogida por las pacientes de ambos grupos, fue la número 1 y para el indicador ideación suicida la alternativa que mostró una mayor tendencia fue la número 3 en ambos grupos de pacientes.

La presencia de síntomas depresivos obtenidos por la aplicación de la EPDS evidenció que el síntoma disforia se observó en el $10 \%$ de las primigestas y en el $8 \%$ de las multíparas, la ansiedad en el $78 \%$ de las primigestas y $70 \%$ de las multíparas, el sentimiento de culpa en el 52 y $56 \%$ de las primigestas y multíparas, la dificultad para la concentración se observo en el $58 \%$ de las primigestas y en el $44 \%$ de las multíparas y la ideación suicida solo se observo en el $6 \%$ de las primigestas y en el $10 \%$ de las multíparas, la significancia estadística que relacionó la misma variable para dos muestras independientes evidenció que solo la ideación suicida mostró diferencias estadísticamente significativas entre las frecuencias del síntoma entre los grupos (Tabla III).

En la Tabla IV se presenta el resultado de la EPDS aplicada a las puérperas, se obtuvo un mínimo de 0 puntos y un máximo de 25 , con un promedio de 15,7 $\pm 2,17$ puntos para primigestas y $16,0 \pm$ 1,94 puntos para multíparas. En la evaluación de la intensidad de los síntomas depresivos se observó que en el grupo de primigestas el $8 \%$ no mostraron riesgo de depresión posparto. El $2 \%$ de las primigestas y $8 \%$ de las multíparas presentaron riesgo límite de depresión posparto y el $90 \%$ y $92 \%$ de ambos grupos presentaron puntajes que indican probable depresión, diferencias no significativas para la presencia de depresión posparto entre primigestas y multíparas.

El $90 \%$ de las primigestas fueron positivas para riesgo de depresión postparto y el $92 \%$ de las multíparas también, así mismo se observó que según DSM IV se evidenció que fueron finalmente diagnosticadas con depresión posparto el $20 \%$ de las primigestas y el $24 \%$ de las multíparas (Tabla V). Considerando los resultados con la aplicación del DSM IV, se puede establecer que la prevalencia total de depresión posparto fue del $22 \%$; asimismo se pudo establecer para esta escala un $90 \%$ de Sensibilidad, $11 \%$ de Especificidad, Valor Predictivo Positivo del $22 \%$, Valor Predictivo Negativo del $81 \%$, Razón de Verosimilitudes Positiva de 1,01 y Razón de Verosimilitudes Negativa de 0,90. 
Tabla II

ANÁLISIS DESCRIPTIVO DE LA ESCALA DE EDIMBURGO POR ÍTEMS EN PRIMIGESTAS Y MULTÍPARAS

\begin{tabular}{|c|c|c|c|c|c|c|c|c|c|c|c|}
\hline \multirow[t]{2}{*}{$\begin{array}{l}\text { Escala de } \\
\text { Edimburgo }\end{array}$} & \multirow[t]{2}{*}{$\begin{array}{l}\text { Contenido } \\
\text { requerido del } \\
\text { ítem }\end{array}$} & \multirow[t]{2}{*}{$\begin{array}{c}\text { Primigestas } \\
X \pm D E\end{array}$} & \multirow[t]{2}{*}{$\begin{array}{c}\text { Multíparas } \\
X \pm D E\end{array}$} & \multicolumn{4}{|c|}{$\begin{array}{c}\text { Primigestas } \\
\text { Distribución } \\
\text { porcentual del ítem } \\
(\%)\end{array}$} & \multicolumn{4}{|c|}{$\begin{array}{c}\text { Multíparas } \\
\text { Distribución } \\
\text { porcentual del ítem } \\
\text { (\%) }\end{array}$} \\
\hline & & & & 0 & 1 & 2 & 3 & 0 & 1 & 2 & 3 \\
\hline \multirow{5}{*}{ Disforia } & $\begin{array}{l}\text { 1.- He sido } \\
\text { capaz de reírme } \\
\text { y ver el lado } \\
\text { divertido de las } \\
\text { cosas. }\end{array}$ & $0,2 \pm 0,3$ & $0,08 \pm 0,3$ & 82 & 18 & 0 & 0 & 92 & 8 & 0 & 0 \\
\hline & $\begin{array}{l}\text { 2.- He disfrutado } \\
\text { mirar hacia } \\
\text { adelante. }\end{array}$ & $0,1 \pm 0,5$ & $0,04 \pm 0,2$ & 94 & 0 & 6 & 0 & 96 & 4 & 0 & 0 \\
\hline & $\begin{array}{l}\text { 7.- Me sentido } \\
\text { tan desdichada } \\
\text { que he tenido } \\
\text { dificultades para } \\
\text { dormir. }\end{array}$ & $2,7 \pm 1,0$ & $2,4 \pm 0,9$ & 10 & 10 & 0 & 80 & 0 & 26 & 6 & 68 \\
\hline & $\begin{array}{l}\text { 8.- Me sentido } \\
\text { triste o } \\
\text { desgraciada. }\end{array}$ & $2,3+1,0$ & $2,3 \pm 0,8$ & 6 & 16 & 18 & 60 & 4 & 12 & 36 & 48 \\
\hline & $\begin{array}{l}\text { 9.- Me he } \\
\text { sentido tan } \\
\text { desdichada que } \\
\text { he estado } \\
\text { llorando. }\end{array}$ & $2,5+0,6$ & $2,1 \pm 0,9$ & 0 & 10 & 22 & 68 & 8 & 8 & 44 & 40 \\
\hline \multirow{2}{*}{ Ansiedad } & $\begin{array}{l}\text { 4.- He estado } \\
\text { nerviosa o } \\
\text { inquieta sin } \\
\text { tener motivo. }\end{array}$ & $1,18 \pm 1,0$ & $1,1 \pm 1,0$ & 40 & 6 & 50 & 4 & 44 & 0 & 56 & 0 \\
\hline & $\begin{array}{l}\text { 5.- He sentido } \\
\text { miedo o he } \\
\text { estado } \\
\text { asustadiza sin } \\
\text { tener motivo. }\end{array}$ & $2,4 \pm 0,9$ & $2,28 \pm 1,0$ & 4 & 18 & 6 & 72 & 4 & 28 & 4 & 64 \\
\hline $\begin{array}{l}\text { Sentimiento } \\
\text { de culpa }\end{array}$ & $\begin{array}{l}\text { 3.- Cuando las } \\
\text { cosas han salido } \\
\text { mal, me he } \\
\text { culpado a mi } \\
\text { misma } \\
\text { innecesariamente. }\end{array}$ & $1,7 \pm 1,1$ & $1,7 \pm 0,9$ & 10 & 50 & 0 & 40 & 0 & 66 & 0 & 34 \\
\hline $\begin{array}{l}\text { Dificultad de } \\
\text { concentración }\end{array}$ & $\begin{array}{l}\text { 6.- Las cosas } \\
\text { me han estado } \\
\text { abrumando. }\end{array}$ & $1,5 \pm 0,9$ & $1,5 \pm 0,8$ & 10 & 44 & 26 & 20 & 4 & 52 & 30 & 14 \\
\hline $\begin{array}{l}\text { Ideación } \\
\text { suicida }\end{array}$ & $\begin{array}{l}\text { 10.- Se me ha } \\
\text { ocurrido la idea } \\
\text { de hacerme } \\
\text { daño. }\end{array}$ & $2,6 \pm 0,6$ & $2,8 \pm 0,7$ & 0 & 10 & 4 & 86 & 4 & 4 & 0 & 92 \\
\hline
\end{tabular}

X \pm DE: Promedio \pm Desvío Estándar 
Tabla III

SÍNTOMAS DEPRESIVOS SEGÚN LA ESCALA DE EDIMBURGO

\begin{tabular}{lccccc}
\hline Síntomas depresivos & \multicolumn{3}{c}{ Primigestas } & \multicolumn{3}{c}{ Multíparas } & Valor p \\
& $\mathrm{n}$ & $\%$ & $\mathrm{n}$ & $\%$ & \\
\hline Disforia & 5 & 10 & 4 & 8 & $0,236^{*}$ \\
Ansiedad & 39 & 78 & 35 & 70 & $0,632^{*}$ \\
Sentimiento de culpa & 26 & 52 & 28 & 56 & $0,869^{*}$ \\
Dificultad para la concentración & 29 & 58 & 22 & 44 & $0,226^{*}$ \\
ldeación suicida & 3 & 6 & 5 & 10 & 0,014 \\
\hline
\end{tabular}

*No significativo

Tabla IV

INTENSIDAD DE LOS SÍNTOMAS DEPRESIVOS (EPDS) EN PRIMIGESTAS Y MULTÍPARAS

\begin{tabular}{|c|c|c|c|c|c|}
\hline \multirow[t]{2}{*}{ Intensidad de los síntomas deprsivos } & \multicolumn{2}{|c|}{ Primigestas } & \multicolumn{2}{|c|}{ Multíparas } & \multirow[t]{2}{*}{ Valor $p$} \\
\hline & $\mathrm{n}$ & $\%$ & $\mathrm{n}$ & $\%$ & \\
\hline$<10$ puntos & 4 & 8 & 0 & 0 & - \\
\hline $10-12$ puntos & 1 & 2 & 4 & 8 & - \\
\hline$\geq 13$ puntos & 45 & 90 & 46 & 92 & $0,280^{*}$ \\
\hline Total & 50 & 100 & 50 & 100 & - \\
\hline Puntuación EPDS & \multicolumn{2}{|c|}{$15,68 \pm 2,17$} & \multicolumn{2}{|c|}{$16,04 \pm 1,94$} & $0,137^{\star}$ \\
\hline
\end{tabular}

* No significativo. EPDS: Escala de Edimburgo para Depresión Posparto

Tabla V

ANÁLISIS DESCRIPTIVO POR EPDS Y DSM IV EN PRIMIGESTAS Y MULTÍPARAS

\begin{tabular}{lcccccccc}
\hline Características de la muestra & \multicolumn{4}{c}{ EPDS } & & \multicolumn{2}{c}{ DSM IV } & - \\
& $\mathrm{n}$ & $\%$ & $\mathrm{n}$ & $\%$ & $\mathrm{n}$ & $\%$ & $\mathrm{n}$ & $\%$ \\
\hline Primigestas & 45 & 90 & 5 & 10 & 10 & 20 & 40 & 80 \\
Multíparas & 46 & 92 & 4 & 8 & 12 & 24 & 38 & 76 \\
\hline
\end{tabular}

EPDS: Escala de Edimburgo para Depresión Posparto.

DSM IV: Manual de Diagnóstico y Estadísticas de Trastornos Mentales.

Para conocer el efecto de la paridad sobre el riesgo de desarrollar depresión postparto, se realizó una tabla de contingencia $2 \times 2$, la cual sirvió para determinar el riesgo de presentar esta patología de acuerdo con la paridad (Tabla VI). Se estableció un OR (IC95\%) de 0,79 (0,3064-2,046), no encontrándose asociación estadísticamente significativa entre paridad y depresión postparto $(p=0,939)$.

\section{DISCUSIÓN}

Los desordenes afectivos ocurren frecuente- mente durante el periodo posparto, la depresión postparto puede ocurrir después de algún tiempo, pero más frecuentemente se presenta de la segunda a la tercera semana después del parto y puede durar hasta un año (13); si bien el cuadro clínico es similar al de los episodios depresivos observados en otras etapas de la vida posee características peculiares que justifican su consideración como entidad especifica $(4,5)$.

Su mecanismo fisiopatológico parece evocar dos mecanismos fundamentales de la personalidad de la gestante y puérpera para defenderse de la ansiedad 


\section{Tabla VI \\ RIESGO DE DEPRESIÓN POSPARTO SEGÚN LA PARIDAD}

\begin{tabular}{lccc}
\hline Paridad & \multicolumn{2}{c}{$\begin{array}{c}\text { Depresión posparto } \\
\text { Positivo }\end{array}$} & Totales \\
\hline Primigestas & 10 & 40 & 50 \\
Multíparas & 12 & 38 & 50 \\
Total & 22 & 78 & 100 \\
\hline
\end{tabular}

que le produce la nueva situación de maternidad, los cuales son la regresión y la introversión (14). Aunque se conoce que durante el puerperio, tanto los cambios bioquímicos existentes así como el estrés (15), pueden desencadenar estos trastornos; no hay evidencia de que un desbalance hormonal o de neurotransmisores sea la causa de la depresión posparto, sin embargo, las teorías más acertadas hasta el momento son las que se relacionan con los cambios hormonales y las susceptibilidades específicas de estos pacientes durante este periodo (11).

Los resultados obtenidos en estas mujeres, las cuales fueron evaluadas con la EPDS cerca de la quinta semana de puerperio, demuestran un riesgo elevado de depresión postparto tanto en multíparas como primíparas, con pruebas de tamizaje positivo en alrededor del $90 \%$; teniendo en consideración que no se trata de un método diagnóstico, sino de rastreo; sus resultados deben ser comparados con una prueba más específica para obtener un diagnóstico certero de la patología. Así, al compararse con los resultados del DSM-IV se obtuvo una prevalencia del $22 \%$; cifra similar a las encontradas por otros autores en la literatura internacional (16), lo cual hace patente el grave problema de subdiagnóstico observado en instituciones de asistencia materno-infantil, principalmente en aquellas como la estudiada donde no se aplica ningún test de rastreo durante el período puerperal.

La Escala de Edimburgo ha mostrado ser un buen método de pesquisa precoz de la depresión posparto, puesto que la mayoría de las madres con test positivos, al aplicárseles la encuesta diagnóstica para depresión según DSM-IV se les ha confirmado el diagnóstico de depresión; es así que esta pesquisa debiera realizarse en dos etapas: el rastreo con un test de autoaplicación, tipo Escala de Edimburgo, seguido de una entrevista diagnóstica con especialistas, para los casos con test positivos (4). Asimismo, estudios realizados en poblaciones de países vecinos, con características sociodemográficas y culturales similares a los de la población zuliana, han evidenciado que este instrumento es confiable y válido para la identificación de posibles síntomas de depresión mayor en las mujeres puérperas (8).

No obstante, en esta investigación si bien el instrumento mostró una alta sensibilidad $(90 \%)$ similar a la reportada por otros autores $(4,5)$, resulto ser poco específico (11\%). Asimismo, esta prueba evidenció un bajo poder predictivo positivo (22\%), mucho menor que el encontrado en otros estudios donde ha sido cercano al $75 \%$ (5).

Como pudo verse, la población del estudio tuvo un mayor porcentaje de mujeres quienes reportaron una prueba positiva en el EPDS, resultados similares a los obtenidos por Morris y cols (13), quienes encontraron un número considerable de pruebas positivas, lo cual fue relacionado al menor estado socioeconómico de la muestra evaluada. Sin embargo, estos resultados difieren de los encontrados tanto por Moreno y cols (5), como Pérez y cols (4), en serie realizadas en Brasil y Chile respectivamente, donde la frecuencia de test positivo según esta escala en ambos estudios fue de $22 \%$; así como del $29 \%$ de incidencia de depresión según EPDS publicada por Wójcik y cols (17).

En relación a los síntomas depresivos evaluados según la EPDS, se encontró una alta prevalencia de ansiedad, sentimientos de culpa y dificultad para la concentración en ambos grupos; sin embargo sólo la ideación suicida mostró una diferencia estadísticamente significativa siendo más frecuente en el grupo de las multíparas. Algunos estudios han señalado que la intensidad de la sintomatología en pacientes con depresión postparto está relacionada con la deficiencia de algunos oligoelementos, sobre todo con el déficit de zinc (17).

También se ha sugerido que el número de partos es un factor asociado a la depresión posnatal, diversas investigaciones han concluido que el nacimiento del primer hijo representa un estrés único y se correlaciona con la depresión de manera más fuerte, que en el caso del segundo o el tercer parto (1). Sin embargo, al igual que los resultados obtenidos por Moreno y cols (5), Evans y cols (10) y Latorre y cols (9), en este estudio la primiparidad no mostró ser un factor determinante para la presencia de depresión posparto, observándose similares puntuaciones entre las primigestas y las multíparas; lo que indica que un instrumento de rastreo como el EPDS debería ser aplicado a todas las puérperas de forma universal y no restringirlo a poblaciones de supuesto mayor riesgo.

Los resultados obtenidos demuestran que en la población del estudio, independientemente de la paridad, la presencia de depresión posparto fue importante desde el punto de vista porcentual en función del valor total de la muestra, de allí la importancia de considerar esta entidad clínica durante la 
evaluación prenatal, por el riesgo de que se presenten síntomas depresivos persistentes después de seis meses de posparto. Así mismo el diagnóstico durante el embarazo puede evitar que esta entidad incida sobre el temperamento del recién nacido, el bajo nivel educativo de la madre y anomalías o enfermedades del hijo o de la madre (11).

Estos resultados sugieren la importancia de identificar los factores de riesgo involucrados en la génesis de depresión posparto. Según Pérez y cols (4), la actitud negativa hacia el embarazo, los antecedentes de patología en el embarazo, parto por cesárea, ansiedad y depresión prenatal, antecedentes personales o familiares de depresión mayor y el embarazo no deseado, son factores que pueden desencadenar una depresión posparto. De igual forma deben considerarse otros factores que pueden tener influencia sobre la aparición de depresión puerperal como las edades extremas, antecedentes de tensión premenstrual, tipo de trabajo, nivel sociocultural, expectativas personales, complicaciones del parto, lactancia materna, grado de apoyo social y emocional por parte de sus allegados, y la mala relación entre la pareja (14).

De igual manera, algunos estudios han señalan una relación entre la depresión y algunos procesos mórbidos asociados a la gestación, evidenciándose una mayor prevalencia de depresión entre las madres que tuvieron neonatos pretérminos o con bajo peso para su edad gestacional (18), así como en aquellas embarazadas que han presentado preeclampsia, en las que se observa un mayor grado de hipocondría y altos niveles de depresión desde antes del nacimiento inclusive (19). Igualmente, la lactancia materna ha demostrado ser un factor protector contra el desarrollo de depresión puerperal, puesto que los niveles de prolactina forman una curva inversa a la de los niveles de estrógenos y progesterona durante el periodo posparto, por lo que razonablemente se ha asumido que las madres que no amamantan a sus bebés, producen un rápido declive en su prolactina y podrían tener una mayor incidencia de depresión posparto (1).

Una de las principales limitaciones del estudio es que incluyó un tamaño de muestreo pequeño, que puede no haber representado adecuadamente a la población total de madres puérperas. Sin embargo, a pesar de no contar en la institución donde fue realizada esta investigación con una estructura para la atención posparto, se logró obtener una muestra, que si bien fue intencionada y no probabilística, estuvo representada por sujetos con características diversas, lo cual permitiría especular que el índice de un tamiz positivo para depresión posparto, podría ser sumamente frecuente en la totalidad de puérpe- ras atendidas en este centro hospitalario.

Asimismo, otra limitante fue no haber incluido tanto a las adolescentes como a las embarazadas añosas dentro del estudio. Al respecto, en la literatura revisada se han encontrado discrepancias en cuanto a la edad como factor de riesgo para el desarrollo de esta patología; se ha señalado que las adolescentes presentan más depresión que las mujeres mayores, puesto que ciertos factores desmoralizadores en el entorno social y las experiencias pasadas, tornan al adolescente más vulnerable a hechos vitales como el embarazo (20), reportándose tasas de prevalencias dentro de este grupo etario de un $14 \%$ a un $48 \%$ (18). Sin embargo, en otros trabajos esta diferencia no ha resultado ser estadísticamente significativa $(4,9,10)$.

Asimismo, otros autores han expresado que como la prevalencia de depresión mayor aumenta con la edad, en las mujeres adultas pudiese ser mayor la frecuencia de depresión posparto debido a que se pudiese tratar de recaídas de una depresión de base, desencadenada por los cambios hormonales productos del parto y los factores socio-culturales relacionados con la llegada de un nuevo hijo (9).

Como lo enuncian los datos presentados, la depresión posparto es una patología de presentación frecuente en las mujeres investigadas, con una frecuencia similar a lo enunciado por la literatura internacional, mostrando una prevalencia de test de rastreo positivo sorprendentemente alta para un país como Venezuela donde el diagnóstico de depresión posparto es realizado con tan poca frecuencia. Por tanto, la detección de depresión posparto es de suma importancia puesto que el no realizar un tratamiento específico durante este periodo, puede acarrear grandes consecuencias tanto para la madre, como para el hijo y la familia en general (11); por lo que se hace imprescindible fomentar la creación de una consulta posparto y la difusión del uso de este tipo de herramientas para disminuir las consecuencias de esta patología.

El uso de una herramienta de sondeo de depresión auto administrada tal como la EPDS puede ser un modo eficiente para mejorar la pesquisa de depresión posparto, sobre todo para los proveedores del servicio médico público donde las limitación de recursos, la sobrecarga de pacientes y la restricción del tiempo para la evaluación médica es más frecuente. La mayoría de las investigaciones efectuadas han demostrado un problema de subdiagnóstico de esta morbilidad y la necesidad de realizar una detección precoz durante el período posparto, dirigido a toda la población materna $(4,5,10,17)$. 


\section{CONCLUSIÓN}

La EPDS demostró ser un instrumento altamente sensible para el diagnóstico de depresión posparto, sin embargo su especificidad y valor predictivo positivo fueron más bajos que los señalados en la literatura internacional, por lo que se debe confirmar el diagnóstico con otras pruebas como la DSM-IV. No existen diferencias significativas en la prevalencia de depresión posparto en los grupos estudiados, salvo para la mayor frecuencia de ideación suicida en multíparas. Se recomienda incluir dentro de la evaluación posnatal la aplicación de estos instrumentos, para detectar oportunamente síntomas depresivos durante el puerperio para evitar sus consecuencias en la madre e hijos.

\section{BIBLIOGRAFÍA}

1. Vargas JE; García M. Depresión post-parto: presencia y manejo psicológico. Centro Regional de Investigación en Psicología. 2009;3(1):11-8.

2. Bowen A, Muhajarine N. Prevalence of antenatal depression in women enrolled in an outreach program in Canada. J Obstet Gynecol Neonatal Nurs 2006;35 (4):491-8.

3. Guadarrama L, Escobar A, Zhang L. Bases neuroquímicas y neuroanatómicas de la depresión. Rev Fac Med UNAM 2006;49(2):66 - 72.

4. Pérez R, Sáez K, Alarcón L, Avilés V, Braganza I, Coleman J. Variables posiblemente asociadas a depresión posparto, según escala Edimburgo. Rev Obstet Ginecol Venez 2007;67(3):187-91.

5. Moreno A, Domíngues L, França PS. Depresión posparto: prevalencia de test de rastreo positivo en puérperas del Hospital Universitario de Brasilia, Brasil. Rev Chil Obstet Ginecol 2004;69(3):209-13.

6. Alvarado R, Rojas M, Monardes J, Perucca E, Neves E, Olea $\mathrm{E}$, et al. Cuadros depresivos en el posparto en una cohorte de embarazadas: construcción de un modelo causal. Rev Chil Neuro-Psiquiat 2000;38(2):84-93.

7. Marchesi C, Bertoni S, Maggini C. Major and minor depression in pregnancy. Obstet Gynecol 2009;113:1292-8.

8. Campo A, Ayola C, Peinado HM, Amor M, Cogollo Z. Escala de Edinburgh para depresión postparto: consistencia interna y estructura factorial en mujeres embarazadas de Cartagena, Colombia. Rev Coloma Obstet Ginecol 2007;58(4):277-83.

9. Latorre JF, Contreras LM, García S, Medina JA. La depresión postparto en madres adolescentes de Bucaramanga, Colombia. Rev Colomb Obstet Ginecol 2006;57(3):156-62.

10. Evans G, Vicuña M, Marín R. Depresión posparto realidad en el sistema público de atención de salud. Rev Chil Obstet Ginecol 2003;68(6):491-4.

11. Orejarena SJ. Trastornos afectivos posparto. MedUNAB 2004;7(20):134-9.

12. Forrest GC, Standish E, Baum JD. Support after perinatal death: a study of support and counselling after perinatal bereavement. $\mathrm{Br}$ Med $\mathrm{J}$ (Clin Res Ed) 1982;285(6353):1475-9.

13. Morris JK, Comerford M, Bernstein PS. Screening for postpartum depression in an inner-city population. Am J Obstet Gynecol 2003;1881217-9.

14. Carrera ML. Papel del obstetra ante la pérdida perinatal. Valencia, España: Universidad de Valencia. Serie de Publicaciones. 2004.

15. Alonso SJ, Damas C, Navarro E. Behavioral despair in mice after prenatal stress. J Physiol Biochem 2000;56(2):77-82.

16. Gjerdingen D, Crow S, McGovern P, Miner M, Center B. Postpartum depression screening at well-child visits: validity of a 2-questions screen and the PHQ-9. Ann Fam Med 2009;7(1):63-70.

17. Wójcik J, Dudek D, Schlegel M, Grabowska M, Marcinek A, Florek E, Piekoszewski W, et al. Antepartum/ postpartum depressive symptoms and serum zinc and magnesium levels. Pharmacological Reports 2006;58:571-6.

18. Kabir K, Sheeder J, Stevens-Simon C. Depression, weight gain, and low birth weight adolescent delivery: Do somatic symptoms strengthen or weaken the relationship? J Pediatr Adolesc Gynecol 2008;21:335-42.

19. Vinaccia S, Sánchez MV, Bustamante EM, Cano EJ, Tobón S. Conducta de enfermedad y niveles de depresión en mujeres embarazadas con diagnóstico de preeclampsia. Int $\mathrm{J}$ Clin Health Psychology 2006;6(1):41-51.

20. Bulnes MS, Ponce CR, Huerta RE, Elizalde R, Santiváñez WR, Aliaga JR, et al. Ajuste psicosocial y estado depresivo en adolescentes de centros escolares de Lima Metropolitana. Revista de Investigación en Psicología 2005;8(2):23-39. 